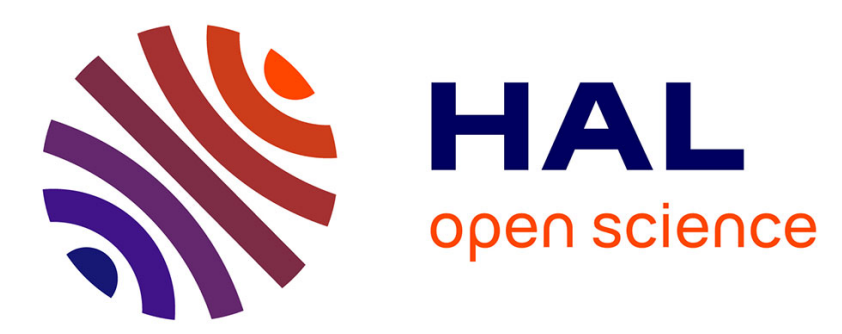

\title{
High-resolution two-dimensional NMR spectra of half-integer-spin quadrupolar nuclei from one-dimensional projections
}

\author{
T. Vosegaard, D. Massiot
}

\section{- To cite this version:}

T. Vosegaard, D. Massiot. High-resolution two-dimensional NMR spectra of half-integer-spin quadrupolar nuclei from one-dimensional projections. Chemical Physics Letters, 2007, 437, pp.120125. 10.1016/j.cplett.2007.01.077 . hal-00127883

\section{HAL Id: hal-00127883 \\ https://hal.science/hal-00127883}

Submitted on 30 Jan 2007

HAL is a multi-disciplinary open access archive for the deposit and dissemination of scientific research documents, whether they are published or not. The documents may come from teaching and research institutions in France or abroad, or from public or private research centers.
L'archive ouverte pluridisciplinaire HAL, est destinée au dépôt et à la diffusion de documents scientifiques de niveau recherche, publiés ou non, émanant des établissements d'enseignement et de recherche français ou étrangers, des laboratoires publics ou privés. 


\title{
High-resolution two-dimensional NMR spectra of half-integer-spin quadrupolar nuclei from one-dimensional projections
}

\author{
Thomas Vosegaard $^{\mathrm{a}, *}$, Dominique Massiot ${ }^{\mathrm{b}}$ \\ ${ }^{a}$ Center for Insoluble Protein Structures (inSPIN), Interdisciplinary Nanoscience Center (iNANO) and Department of \\ Chemistry, University of Aarhus, DK-8000 Aarhus C, Denmark \\ ${ }^{b}$ Centre de Recherche sur les Matériaux à Haute Température, CNRS UPR4212 FR2950, 45071 Orléans Cedex 2, \\ France
}

* Corresponding author:

Thomas Vosegaard

Center for Insoluble Protein Structures (inSPIN),

Interdisciplinary Nanoscience Center (iNANO) and Department of Chemistry

University of Aarhus

Langelandsgade 140, DK-8000 Aarhus C

Denmark

Email tv@,chem.au.dk

Tel $\quad+4589423866$

Mobile +4560202639

Fax $\quad+4586196199$

Key-Words: Projection-Reconstruction; Second-order quadrupole interaction; PRODI; Radial Sampling 


\begin{abstract}
We present a technique providing high-resolution spectra of quadrupolar nuclei with half-integer nuclear spin displaying significant second-order linebroadening. The technique, dubbed chemical shift - quadrupolar projection-reconstruction of one-dimensional spectra (CQ-PRODI) exploits the different magnetic field dependence of the chemical shift and second-order quadrupolar effect. Onedimensional spectra recorded at different magnetic field strengths are represented as projections along different directions in the two-dimensional spectrum that correlates the chemical shift and the second-order quadrupolar lineshape. We present CQ-PRODI spectra for ${ }^{27} \mathrm{Al}$ in $9 \mathrm{Al}_{2} \mathrm{O}_{3} \cdot 2 \mathrm{~B}_{2} \mathrm{O}_{3}$ and for ${ }^{71} \mathrm{Ga}$ in $\beta-\mathrm{Ga}_{2} \mathrm{O}_{3}$ which display resolution of all sites in the chemical shift dimension.
\end{abstract}




\section{Introduction}

Solid-state nuclear magnetic resonance (NMR) studies on quadrupolar nuclei with half-integer nuclear spin provide a wealth of information contributing to the understanding of a host of compounds ranging from inorganic materials such as ceramics [1] and glasses [2] to biological macromolecules $[3,4]$. One of the major challenges in studying half-integer-spin quadrupolar nuclei is that the typically very strong quadrupole coupling interactions (several $\mathrm{MHz}$ ) of these nuclei will cause a significant line broadening of the NMR resonances. Even for the central transition $(\mathrm{m}=1 / 2$ $\leftrightarrow-1 / 2$ ) which is not broadened by the quadrupole interaction to first order, will be broadened by the second-order quadrupolar effect, a broadening that may only be reduced but not removed by magic-angle spinning (MAS).

The introduction of dynamic-angle spinning (DAS) [5,6] and double-rotation (DOR) [6,7] as well as the more recent multiple-quantum MAS (MQMAS) [8] and satellite-transition MAS (STMAS) [9] techniques have been essential in the past decade's applications of solid-state NMR for structural characterization targeting quadrupolar nuclei since they offer high-resolution spectra by complete removal of the second order lineshape. With these techniques, the high resolution is achieved, but unfortunately often at the expense of very delicate experimental setup (DOR, DAS, and STMAS) or low sensitivity (MQMAS).

A complementary approach to improving the resolution for quadrupolar nuclei displaying second-order broadened resonances is to employ the highest available magnetic field strength since the second-order quadrupolar effect is inversely proportional to the magnetic field. For example, a recent study employing magnets with up to $40 \mathrm{~T}$ field strengths provided complete resolution of 4 ${ }^{27} \mathrm{Al}$ sites in the aluminoborate $9 \mathrm{Al}_{2} \mathrm{O}_{3} \cdot 2 \mathrm{~B}_{2} \mathrm{O}_{3}$ (A9B2) [10], while experiments performed at lower commercially available field strengths give second-order broadened spectra with significant overlap of the resonances. At the extreme magnetic field strength of $40 \mathrm{~T}$ the linewidth due to drift and field inhomogeneity exceeds the second-order linewidth and hence the high resolution is achieved at the expense of the information on the quadrupole coupling interaction. 
In this Letter we demonstrate that it is possible to reconstruct a 2D spectrum correlating the highresolution chemical shift with the second-order quadrupolar lineshape, from 1D spectra recorded at different magnetic field strengths. The technique, dubbed chemical shift - quadrupolar projectionreconstruction of one-dimensional spectra (CQ-PRODI), may be applied to both spinning and static sample with a sensitivity comparable to that of a one-dimensional spectrum.

\section{Experimental}

The ${ }^{27} \mathrm{Al}$ spectra of A9B2 have been acquired under rapid magic-angle spinning (15 to $30 \mathrm{kHz}$ ) using single-pulse excitation with typical recycle delays of $1 \mathrm{~s}$ at magnetic fields of $9.4 \mathrm{~T}$ to $19.5 \mathrm{~T}$ (400 MHz to $830 \mathrm{MHz}{ }^{1} \mathrm{H}$ Larmor frequency) in stable and homogeneous superconducting magnets [11], and at magnetic fields of $25 \mathrm{~T}$ and $40 \mathrm{~T}$ in resistive or hybrid magnets. In the latter case individual free-induction decays (FIDs) were acquired and referenced independently to correct for the field-drift between the scans [10]. The ${ }^{71} \mathrm{Ga}$ NMR spectra of $\beta-\mathrm{Ga}_{2} \mathrm{O}_{3}$ were recorded using fullecho acquisition to obtain pure absorption mode spectra with flat baselines and improved signal-tonoise ratios [12]. All 1D spectra were processed on the spectrometer and, if necessary, corrected for distorted baselines by piecewise spline interpolation of the baseline regions using SIMPSON $[13,14]$. Projection-reconstructions of the 2D CQ-PRODI spectra were also performed using SIMPSON by normalizing all spectra and subsequently following the reconstruction procedure applied for SLF-PRODI [15].

\section{Results and discussion}

--- Figure 1 here ---

Projection-reconstruction techniques have recently been introduced to reduce the dimensionality of a variety of liquid-state $[16,17]$ and solid-state $[15,18]$ NMR experiments. In a more general 
scope we can remark that while most of the multidimensional experiments are acquired by timeconsuming stepwise increment of each evolution time, there exist several examples of reconstruction methods that rely on other degrees of freedom of the system: heteronuclear dipolar scaling factor in SLF-PRODI [15], spinning angle in VACSY [19] or DACSY [20] experiments, pitch encoding in PASS [21] or QPASS [22] experiments, spatial or orientational encoding in onepulse acquisition schemes [23,24], spinning sidebands of MAS spectra in TOP processing [25,26]. We will apply the PRODI technique to quadrupolar nuclei by taking advantage of the fact that the strength of the chemical shift interaction is proportional to the external magnetic field, while the second-order quadrupolar effect is inversely proportional to the external magnetic field. Bearing this in mind, we may regard the spectrum of a quadrupolar nucleus at a given magnetic field as being a projection with a given angle in a $2 \mathrm{D}$ chemical-shift - quadrupolar correlation spectrum. For illustration Fig. 1a shows a series of ${ }^{27} \mathrm{Al}$ spectra of $\mathrm{A} 9 \mathrm{~B} 2$. In Fig. 1b these spectra are represented in the $2 \mathrm{D}$ spectrum, and the scales have been arbitrarily chosen such that the $\mathrm{B}_{0}=17.5$ $\mathrm{T}$ spectrum forms an angle of $45^{\circ}$ with the quadrupolar axes. A spectrum recorded at an other magnetic field strength $\left(\mathrm{B}_{0}{ }^{\prime}\right)$ will be represented by an angle of $\arctan \left(\left(\mathrm{B}_{0}{ }^{\prime} / \mathrm{B}_{0}\right)^{2}\right)$. For example, 9.4$\mathrm{T}$ and 25-T spectra will correspond to projections at angles of $16^{\circ}$ and $63^{\circ}$, respectively, as shown in Fig. 1b. The corresponding projections are shown as contour plots in Fig. 1c. By combining these 2D projections we can reconstruct the 2D CQ-PRODI spectrum providing the desired chemicalshift/quadrupolar correlation. We note that the different field dependence of the chemical shift and second-order quadrupolar effect has previously been exploited in the spectral interpretation, e.g., using a so-called discontinuity multifield graph $[12,27]$ or a second-order graphic extrapolation diagram $[11,28]$. We also note that the TOP representation for half-integer quadrupolar nuclei $[25,26]$ allows a separation of the second-order lineshapes for the central and individual satellite transitions which may be represented as different projections in a CQ-PRODI spectrum.

A9B2 contains one tetrahedrally coordinated $\mathrm{Al}$ site $\left(\mathrm{Al}_{\mathrm{IV}}\right)$, two different penta coordinated $\mathrm{Al}$ sites $\left(\mathrm{Al}_{\mathrm{V}(1)}\right.$ and $\left.\mathrm{Al}_{\mathrm{V}(2)}\right)$, and one octahedrally coordinated $\mathrm{Al}$ site $\left(\mathrm{Al}_{\mathrm{VI}}\right)$ with double occupancy and 
hence double intensity in the NMR spectra [11]. Even at the high field strength of 25 T (Fig. 1a) there is still a slight overlap of $\mathrm{Al}_{\mathrm{IV}}$ with one of the $\mathrm{Al}_{\mathrm{V}}$ sites and a significant overlap between the two $\mathrm{Al}_{\mathrm{V}}$ sites, while a spectrum recorded at $40 \mathrm{~T}$ displays complete resolution of the four sites [10]. The 2D CQ-PRODI spectrum resulting from projections from spectra recorded at six different magnetic field strengths from $9.4 \mathrm{~T}$ to $40 \mathrm{~T}$ is represented in Fig. 1d with the quadrupolar axis corresponding to a 9.4-T spectrum. The spectrum displays the expected four resonances, and the horizontal slices through these resonances (Fig. 1e) show the second-order lineshapes for these four sites. As expected these lineshapes have their leftmost singularity situated close to zero since the horizontal slices are not influenced by the chemical shift of the particular resonance in a CQPRODI spectrum. A comparison of the second-order quadrupolar lineshapes of the four resonances with the lineshapes simulated employing previously determined parameters [11] (Fig. 1f) shows good overall agreement while in particular the two $\mathrm{Al}_{\mathrm{V}}$ sites show distortions. These artefacts appear due to the heavy overlap between these two sites in all spectra displaying a second-order quadrupolar lineshape for these two sites.

The resolution achieved in the chemical shift dimension of the CQ-PRODI spectrum depends on resolution of the 1D spectrum from the highest magnetic field strength used for the reconstruction, and since the 1D 40-T spectrum was employed to achieve the CQ-PRODI spectrum in Fig. 1 it is not surprising that a resolution of all four sites was achieved. To cope with spectra of lower resolution, and in the case of A9B2 to employ spectra only from todays commercially available range of superconducting magnets, we have performed a large number of numerical simulations of CQ-PRODI spectra and have come to the conclusion that the chemical-shift projections are always free of second-order lineshape and only contain a Gaussian/Lorentzian-like lineshape. Being equipped with the chemical shift projection with Gaussian/Lorentzian peaks rather than peaks with second-order quadrupolar lineshapes is interesting, since it opens for the possibility to cautiously apply resolution enhancement techniques to the chemical shift projection. Among many resolution 
enhancement techniques, we have found that the MaxEnt algorithm with multiple search directions [29] as implemented for NMR purposes by Hoch and Stern [30] is the most stable for our purposes.

--- Figure 2 here ---

This procedure is illustrated in Fig. 2 for ${ }^{27} \mathrm{Al}$ in A9B2, and Fig. 2a shows the CQ-PRODI spectrum obtained from 1D spectra at a number of magnetic field strengths from $9.4 \mathrm{~T}$ to $25 \mathrm{~T}$. In this spectrum we observe the presence of four patterns of which those corresponding to the $\mathrm{Al}_{\mathrm{V}}$ sites are heavily overlapping as also observed in the chemical-shift projection in Fig. 2b, and overall this projection does not improve the resolution compared to the 25-T spectrum (Fig. 1a), but as mentioned above the resonances now show a Gaussian/Lorentzian lineshape. Figure $2 \mathrm{~d}$ shows the chemical shift projection (Fig. 2b) after it has been subjected to a MaxEnt deconvolution with a 6ppm Lorentzian point-spread function. We may use the deconvoluted chemical shift projection to improve the resolution in the 2D CQ-PRODI spectrum by adding it as a horizontal projection in a refined reconstruction of the CQ-PRODI spectrum. Figure 2c shows the horizontal projection from the deconvoluted spectrum in Fig. 2d, and the CQ-PRODI spectrum resulting from projections of the 1D spectra recorded at magnetic field strengths between $9.4 \mathrm{~T}$ and $25 \mathrm{~T}$ and horizontal projection is shown in Fig. 2e. This spectrum is comparable to the one in Fig. 1c, and is superior to the resolution of the $25-\mathrm{T}$ spectrum. We furthermore note that the slices through the four resonances display second-order quadrupolar lineshapes (not shown) are virtually identical to those reported in Fig. 1d.

--- Figure 3 here ---

--- Table 1 here --- 
A very useful feature of CQ-PRODI spectra is that their chemical shift projection display resonances at the position of the isotropic chemical shift while regular 1D spectra show displacements from the isotropic chemical shifts by the isotropic second-order quadrupolar shift. Figure 3 shows the $40 \mathrm{~T}^{27} \mathrm{Al}$ spectrum of A9B2 (Fig. 3a) along with the $9.4 \mathrm{~T}$ to $40 \mathrm{~T}$ CQ-PRODI chemical shift projection (Fig. $3 \mathrm{~b}$ and Fig. 1c). Table 1 summarizes the peak positions measured in the $40 \mathrm{~T}$ spectrum and in CQ-PRODI spectra achieved using spectra from magnetic fields of 9.4 T $40 \mathrm{~T}$ and $9.4 \mathrm{~T}-25 \mathrm{~T}$, respectively. The CQ-PRODI values agree very well with previously determined chemical shifts [11,31], while we observe that the resonances in the $40 \mathrm{~T}$ spectrum are shifted by up to $3 \mathrm{ppm}$ due to the second-order quadrupolar shift even if the actual second-order quadrupolar lineshape is masked by the principal field inhomogeneity.

--- Figure 4 here ---

While the MQMAS and STMAS techniques fail for nuclei with large quadrupole couplings due to low sensitivity in the case of MQMAS [32] and excessive line-broadening for STMAS [33], there is no such limitation to CQ-PRODI. To investigate this appealing property of CQ-PRODI we have applied this technique to ${ }^{71} \mathrm{Ga}$ spectra of $\beta-\mathrm{Ga}_{2} \mathrm{O}_{3}$ which contains a GavI and a Ga $a_{\text {IV }}$ site with quadrupole couplings of $8.3 \mathrm{MHz}$ and 11.2 MHz, respectively [12,34]. These values are well beyond the size of the quadrupole couplings amenable to MQMAS or STMAS, and only DAS [27] and single-crystal NMR [34] have achieved complete resolution of the two sites. Even a regular MAS spectrum at $9.4 \mathrm{~T}$ would result in a second-order lineshape with a width exceeding $70 \mathrm{kHz}$ implying that a MAS spectrum acquired using a modest spinning rate would result in overlap of the spinning sidebands $[12,27,35]$ which are very difficult to interpret and to model and would be very difficult to handle with CQ-PRODI. To circumvent this problem we decided to use CQ-PRODI on a series of static ${ }^{71} \mathrm{Ga}$ spectra of $\beta-\mathrm{Ga}_{2} \mathrm{O}_{3}$, recorded at magnetic field strengths of 7.1 T, 9.4T, 11.7 $\mathrm{T}$, and 18.8 $\mathrm{T}$ as illustrated in Fig. 4. The CQ-PRODI reconstruction of the four 1D spectra, shown in 
Fig. 4e, suggests the presence of two sites, but the sum projection along of the chemical shift dimension reveals a strong overlap of the resonances for these sites.

The strong overlap in the CQ-PRODI spectrum is partly ascribed to the larger linewidth of the static second-order lineshape compared to the MAS lineshape. For the MAS lineshape the outer singularities appear at frequencies of $v_{\min }^{\mathrm{MAS}}=-2\left(6+\eta^{2}\right) a$ and $v_{\max }^{\mathrm{MAS}}=-12 / 7(1-\eta)^{2} a$, where $a=3 v_{Q}^{2} / 4 v_{0}$ and $v_{Q}=C_{Q} /(4 I(2 I-1))$. For a static sample these values are $v_{\min }^{\text {Stat }}=-16(1+\eta) a$ and $v_{\max }^{\text {Stat }}=(3+\eta)^{2} a$, and hence it may easily be established that the width of the static secondorder lineshape exceeds that of the MAS lineshape by factors of $2.4-3.4$ depending on the asymmetry parameter, $\eta$, leading to significantly increased overlap in static spectra than in MAS spectra. While this increased overlap is an obvious challenge to CQ-PRODI, the form of the static second-order lineshape allows establishing a horizontal projection. This was not possible for MAS lineshapes which only reside on one side of the zero frequency, but becomes possible for static lineshapes which spread over positive and negative values around the zero frequency. Hence we may use the chemical shift trace at zero frequency in the quadrupolar dimension of the CQ-PRODI spectrum (indicated by a dashed line in Fig. 4e) as a horizontal projection, leading to the improved CQ-PRODI spectrum in Fig. 4f. In this spectrum the appearance of two sites is very clear from the sum projection of the chemical shift dimension which displays significantly improved resolution when compared to the projection in Fig. 4e.

To further improve the resolution of the static CQ-PRODI spectrum, we use the MaxEnt deconvolution strategy and add a horizontal MaxEnt deconvoluted projection of the chemical shift sum projection (Fig. 4f) to yield the final 2D CQ-PRODI spectrum in Fig. 4g. The chemical shift projection from this spectrum displays two resonances located at $29 \pm 33 \mathrm{ppm}\left(\mathrm{Ga}_{\mathrm{VI}}\right)$ and $232 \pm 33$ ppm $\left(\mathrm{Ga}_{\mathrm{IV}}\right)$, respectively, where the error limits represent $1 / 4$ of the FWHH linewidths. These chemical shifts compare reasonably well with our previous high-precision measurements of $25 \pm 1$ ppm and $196 \pm 2$ ppm from single-crystal NMR [34], although the match is not perfect. An obvious reason for this is that the static lineshapes are influenced by the chemical shift anisotropies (CSA) 
which amount to $37 \mathrm{ppm}\left(\mathrm{Ga}_{\mathrm{VI}}\right)$ and $-33 \mathrm{ppm}\left(\mathrm{Ga}_{\mathrm{IV}}\right)$ [34] and thereby add to the linewidths and may shift the resonance position in the chemical shift dimension of the CQ-PRODI spectrum. The CSA broadening in the chemical shift dimension of the CQ-PRODI spectrum will increase proportionally with the magnetic field strength suggesting that at some point there will no longer be a gain in resolution by adding projections from static spectra recorded at higher magnetic fields. This happens when the line broadening from the CSA exceeds that of the second-order quadrupolar lineshape which would at approximately $24 \mathrm{~T}$ for $\mathrm{Ga}_{\mathrm{VI}}$ and $40 \mathrm{~T}$ for $\mathrm{Ga}_{\mathrm{IV}}$.

Most half-integer quadrupolar nuclei display appreciable CSAs so the CSA broadening will be a pronounced problem (of growing importance at higher magnetic fields) for many cases when applying static experiments to reconstruct the CQ-PRODI spectrum. This fact favors the use of MAS experiments for CQ-PRODI even in cases where the second-order lineshape exceeds the spinning frequency. In these cases we suggest the use of infinite spinning speed reconstructions from QPASS experiments [22] which displays the appreciated line narrowing by MAS and furthermore eliminate any contributions from the CSA.

\section{Conclusions}

In conclusion, we have presented the CQ-PRODI technique which employs 1D static or MAS experiments of half-integer quadrupolar nuclei to reconstruct a high-resolution 2D spectrum correlating the chemical shift in one dimension with the second-order quadrupolar lineshape in the other dimension. By applying MaxEnt resolution enhancement in the data processing, CQ-PRODI spectra of considerably better resolution than the original 1D spectra may be achieved. Furthermore, the CQ-PRODI spectra compensate for the second-order quadrupolar shift in the high-resolution dimension allowing determination of isotropic chemical shift. The CQ-PRODI spectra display sensitivities comparable with 1D spectra making CQ-PRODI an obvious candidate for achieving high-resolution spectra of quadrupolar nuclei in samples with low sensitivity. Such cases include 
low- $\gamma$ nuclei and nuclei with low natural abundance of the relevant isotope or samples with low abundance of the nucleus in question, e.g., metals in metal binding sites of proteins.

\section{Acknowledgment}

Support from the Danish National Research Foundation, the Danish Natural Science Research Council, CNRS UPR4212, FR 2950, and ANR RMNHRHC is acknowledged. We would like to thank Prof. Z. Gan and NHMFL Tallahassee, FL, for performing the very-high field experiments at $19.5 \mathrm{~T}, 25 \mathrm{~T}$, and $40 \mathrm{~T}$.

\section{References}

[1] K.J.D. Mackenzie, Brit. Ceram. Trans. 99 (2000) 231.

[2] I. Farnan, P.J. Grandinetti, J.H. Baltisberger, J.F. Stebbins, U. Werner, M.A. Eastman, A. Pines, Nature 358 (1992) 31.

[3] D. Rovnyak, M. Baldus, G. Wu, N.V. Hud, J. Feigon, R.G. Griffin, J. Am. Chem. Soc. 122 (2000) 11423.

[4] V. Lemaitre, M.E. Smith, A. Watts, Solid State Nucl. Magn. Reson. 26 (2004) 215.

[5] A. Llor, J. virlet, Chem Phys Lett 152 (1988) 248.

[6] B.F. Chmelka, K.T. Mueller, A. Pines, J. Stebbins, Y. Wu, J.W. Zwanziger, Nature 339 (1989) 42.

[7] A. Samoson, E. Lippmaa, A. Pines, Mol. Phys. 65 (1988) 1013.

[8] L. Frydman, J.S. Harwood, J. Am. Chem. Soc. 117 (1995) 5367.

[9] Z. Gan, J. Am. Chem. Soc. 122 (2000) 2142.

[10] Z. Gan, P. Gor'kov, T.A. Cross, A. Samoson, D. Massiot, J. Am. Chem. Soc. 124 (2002) 5634.

[11] D. Massiot, D. Muller, T. Hubert, M. Schneider, A.P. Kentgens, B. Cote, J.P. Coutures, W. Gessner, Solid State Nucl. Magn. Reson. 5 (1995) 175.

[12] D. Massiot, I. Farnan, N. Gautier, D. Trumeau, A. Trokiner, J.P. Coutures, Solid State Nucl. Magn. Reson. 4 (1995) 241.

[13] M. Bak, J.T. Rasmussen, N.C. Nielsen, J. Magn. Reson. 147 (2000) 296.

[14] T. Vosegaard, A. Malmendal, N.C. Nielsen, Chemical Monthly 133 (2002) 1555.

[15] K. Bertelsen, J.M. Pedersen, N.C. Nielsen, T. Vosegaard, J. Magn. Reson. in press (2007).

[16] T. Szyperski, D.C. Yeh, D.K. Sukumaran, H.N. Moseley, G.T. Montelione, Proc. Natl. Acad. Sci. 99 (2002) 8009.

[17] E. Kupce, R. Freeman, J. Am. Chem. Soc. 126 (2004) 6429.

[18] N.S. Astrof, C.E. Lyon, R.G. Griffin, J. Magn. Reson. 152 (2001) 303.

[19] L. Frydman, G.C. Chingas, Y.K. Lee, P.J. Grandinetti, M.A. Eastman, G.A. Barrall, A. Pines, J. Chem. Phys. 97 (1992) 4800.

[20] A. Medek, J.R. Sachleben, P. Beverwyk, L. Frydman, J. Chem. Phys. 104 (1996) 5374.

[21] O.N. Antzutkin, S.C. Shekar, M.H. Levitt, J. Magn. Reson. A 115 (1995) 7.

[22] D. Massiot, V. Montouillout, F. Fayon, P. Florian, C. Bessada, Chem. Phys. Lett. 272 (1997) 295.

[23] L. Frydman, T. Scherf, A. Lupulescu, Proc. Natl. Acad. Sci. 99 (2002) 15858. 
[24] R. Bhattacharyya, L. Frydman, Ultrafast Solid-State 2D NMR Experiments via Orientational Encoding, 2006, p. 16014.

[25] P. Blümler, B. Blümich, J. Jansen, Solid State Nucl. Magn. Reson. 1 (1992) 111.

[26] D. Massiot, J. Hiet, N. Pellerin, F. Fayon, M. Deschamps, S. Steuernagel, P.J. Grandinetti, J. Magn. Reson. 181 (2006) 310.

[27] D. Massiot, I. Farnan, N. Gauthier, D. Trumeau, P. Florian, P.J. Grandinetti, J. Chim. Phys. 92 (1995) 1847.

[28] K.T. Mueller, J.H. Baltisberger, E.W. Wooten, A. Pines, J. Phys. Chem. 96 (1992) 7001.

[29] J. Skilling, R. Bryan, Mon. Not. R. Astron. Soc. 211 (1984) 111.

[30] J.C. Hoch, A.S. Stern: NMR data processing, John Wiley \& Sons, Hoboken, New Jersey, 1996.

[31] T. Vosegaard, D. Massiot, P.J. Grandinetti, Chem. Phys. Lett. 326 (2000) 454.

[32] L. Frydman, in D.M. Grant, R.K. Harris (Eds.), Encyclopedia of Nuclear Magnetic Resonance. J. Wiley \& Sons, Chichester, 2002, p. 262.

[33] Z. Gan, P. Srinivasan, J.R. Quine, S. Steuernagel, B. Knott, Chem. Phys. Lett. 367 (2003) 163.

[34] T. Vosegaard, I.P. Byriel, L. Binet, D. Massiot, H.J. Jakobsen, J. Am. Chem. Soc. 120 (1998) 8184.

[35] D. Massiot, T. Vosegaard, N. Magneron, D. Trumeau, V. Montouillout, P. Berthet, T. Loiseau, B. Bujoli, Solid State Nucl. Magn. Reson. 15 (1999) 159.

\section{Table 1}

${ }^{27} \mathrm{Al}$ peak positions in the $40 \mathrm{~T}$ single-pulse spectrum of A9B2 and in the chemical shift projections of the CQ-PRODI spectra.

Peak position (ppm)

\begin{tabular}{lcccccc} 
Spectrum type & Field strength $(\mathrm{T})$ & $\mathrm{Al}_{\mathrm{VI}}$ & $\mathrm{Al}_{\mathrm{V}(1)}$ & $\mathrm{Al}_{\mathrm{V}(2)}$ & $\mathrm{Al}_{\mathrm{IV}}$ & Ref. \\
\hline MAS $^{\mathrm{a}}$ & 40 & $7(2)$ & $51(2)$ & $42(2)$ & $69(3)$ & {$[10]$} \\
CQ-PRODI $^{\mathrm{a}}$ & $9.4-40$ & $8(2)$ & $53(3)$ & $44(3)$ & $72(3)$ & This work \\
CQ-PRODI $^{\mathrm{a}}$ & $9.4-25$ & $8(2)$ & $52(3)$ & $40(3)$ & $75(4)$ & This work \\
DOR / MAS $^{\text {MQMAS }}$ & $7-17.6$ & 9 & 52 & 44 & 71 & {$[11]$} \\
& $7.1-9.4$ & $8(1)$ & $52(1)$ & $44(1)$ & $71(1)$ & {$[31]$}
\end{tabular}

${ }^{\mathrm{a}}$ The numbers in parentheses represent the half width at half height, reflecting accuracy of the measured peak position. 


\section{Figure captions}

Figure 1. (a) 27Al MAS NMR spectra of A9B2 and (b) schematic representation of a twodimensional chemical shift - quadrupolar correlation spectrum showing the ${ }^{27} \mathrm{Al}$ spectra from (a) at orientations according to their magnetic field strengths. (c) Corresponding 2D projections in a 17.5 T chemical shift - quadrupolar correlation spectrum. (d) 2D 9.4-T CQ-PRODI spectrum achieved from projections from spectra recorded at $9.4 \mathrm{~T}, 14.1 \mathrm{~T}, 17.6 \mathrm{~T}, 19.5 \mathrm{~T}, 25 \mathrm{~T}$, and $40 \mathrm{~T}$, and (e) traces through the four resonances in the chemical shift dimension. (f) Simulated second-order lineshapes based on previously reported parameters [11,31]. Contour levels are drawn at 10\%, 20\%, $\ldots, 90 \%$ of the maximum intensity.

Figure 2. ${ }^{27} \mathrm{Al}$ CQ-PRODI spectrum for A9B2 (a) and corresponding chemical shift projection (b) achieved using projections from spectra recorded at 9.4 T, 14.1 T, 17.6 T, 19.5 T, and 25 T. MaxEnt resolution enhanced chemical-shift spectrum (d) and the corresponding projection (c). CQ-PRODI spectrum resulting from the 1D spectra horizontal projection (e) corresponding chemical shift projection (f). Contour levels are drawn at $10 \%, 20 \%, \ldots, 90 \%$ of the maximum intensity.

Figure 3. (a) $40 \mathrm{~T}^{27} \mathrm{Al}$ spectrum and (b) chemical-shift projection of the ${ }^{27} \mathrm{Al} \mathrm{CQ}-\mathrm{PRODI}$ spectrum of A9B2. The projection is identical to the vertical projection in Fig. 1d. The dashed lines indicate the isotropic chemical shifts for the four Al sites.

Figure 4. Static ${ }^{71} \mathrm{Ga}$ spectra of $\beta-\mathrm{Ga}_{2} \mathrm{O}_{3}$ recorded at (a) $7.1 \mathrm{~T}$, (b) $9.4 \mathrm{~T}$, (c) $11.7 \mathrm{~T}$, and (d) $18.8 \mathrm{~T}$. (e) 9.4-T CQ-PRODI spectrum obtained from the four 1D spectra, (f) the same spectrum but with an additional horizontal projection resulting from the slice at zero in the quadrupolar dimension as indicated by a dashed line in (e). (g) The same spectrum with an additional horizontal MaxEnt projection. Contour levels are drawn at $10 \%, 20 \%, \ldots, 90 \%$ of the maximum intensity. 

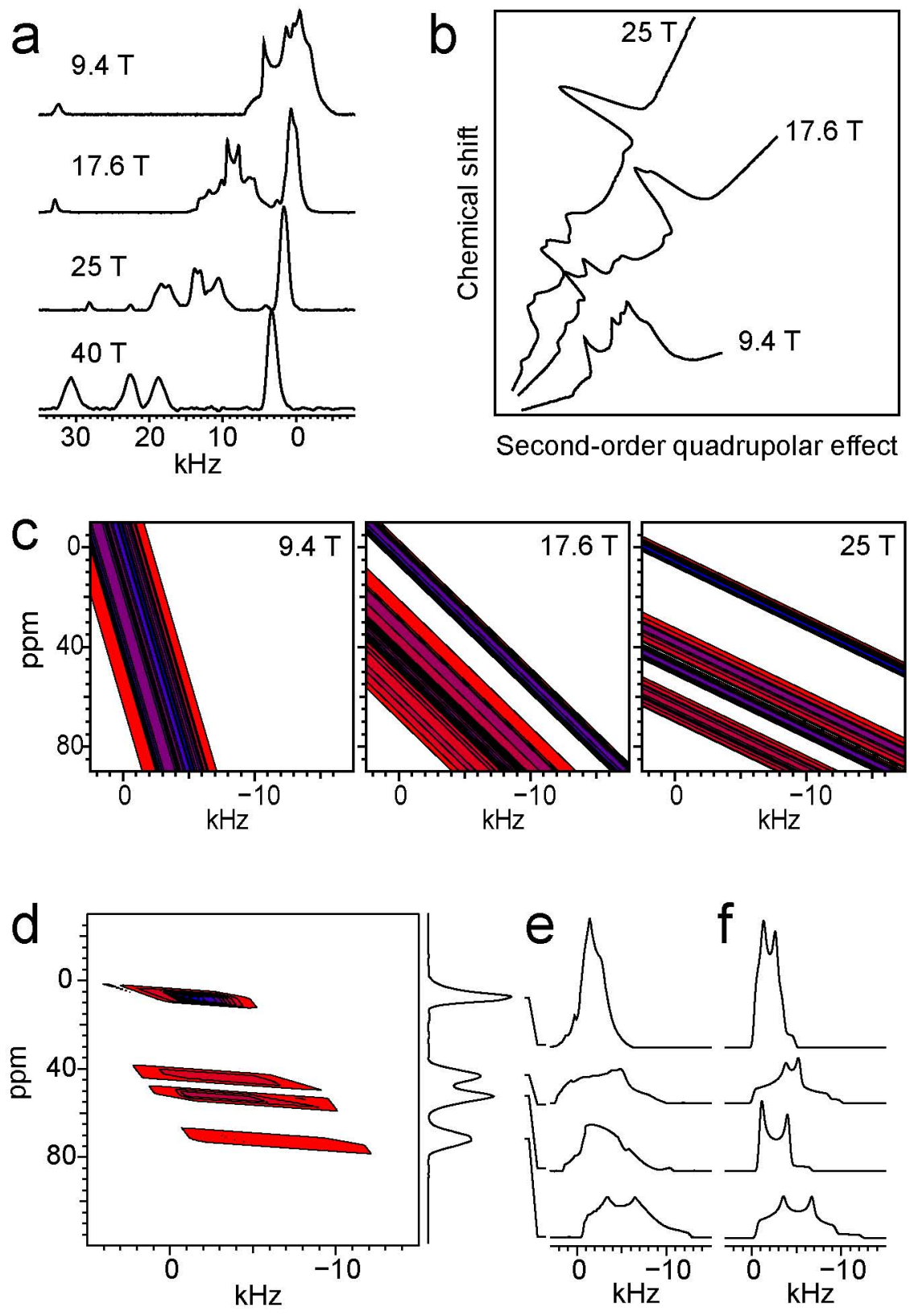
Figure 2

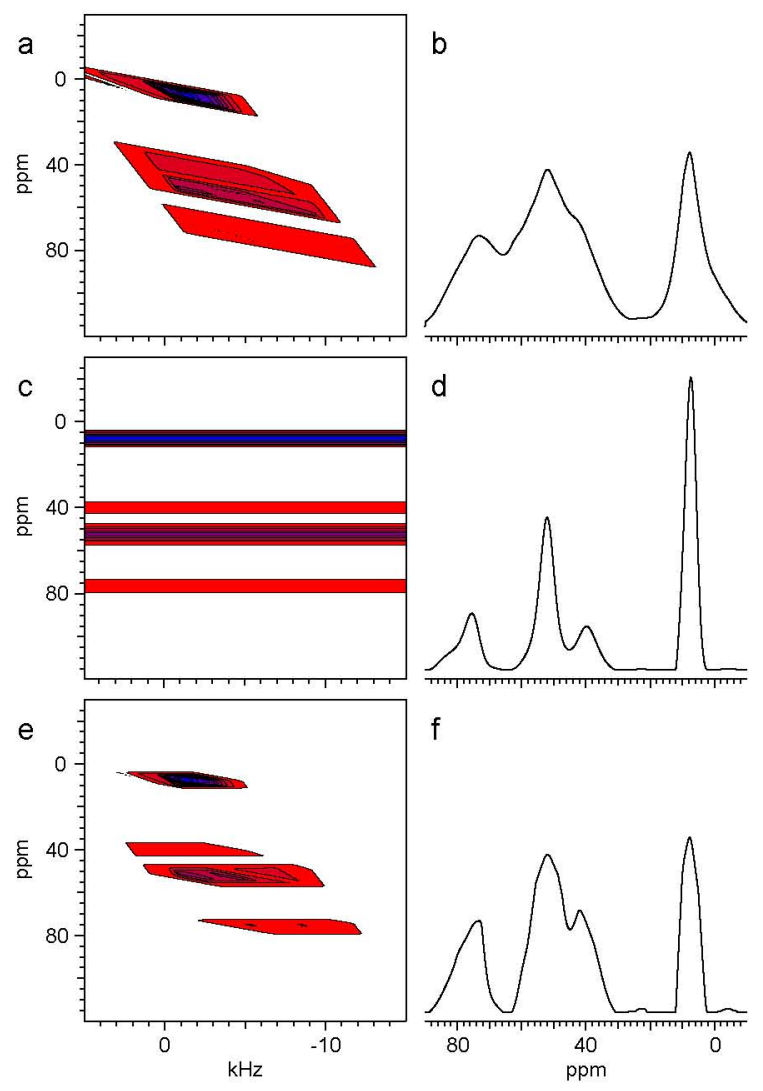


Figure 3

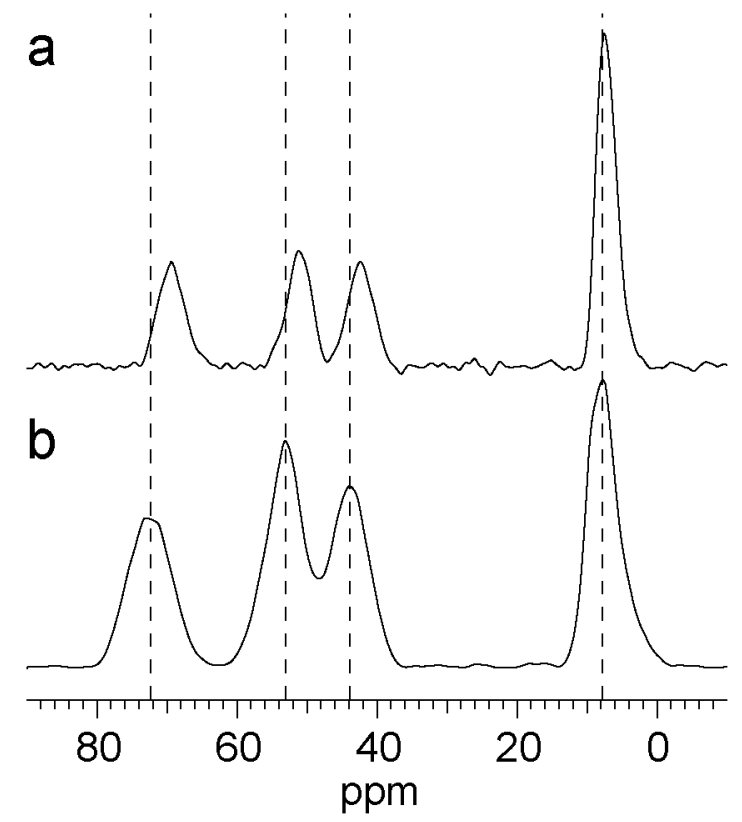


Figure 4

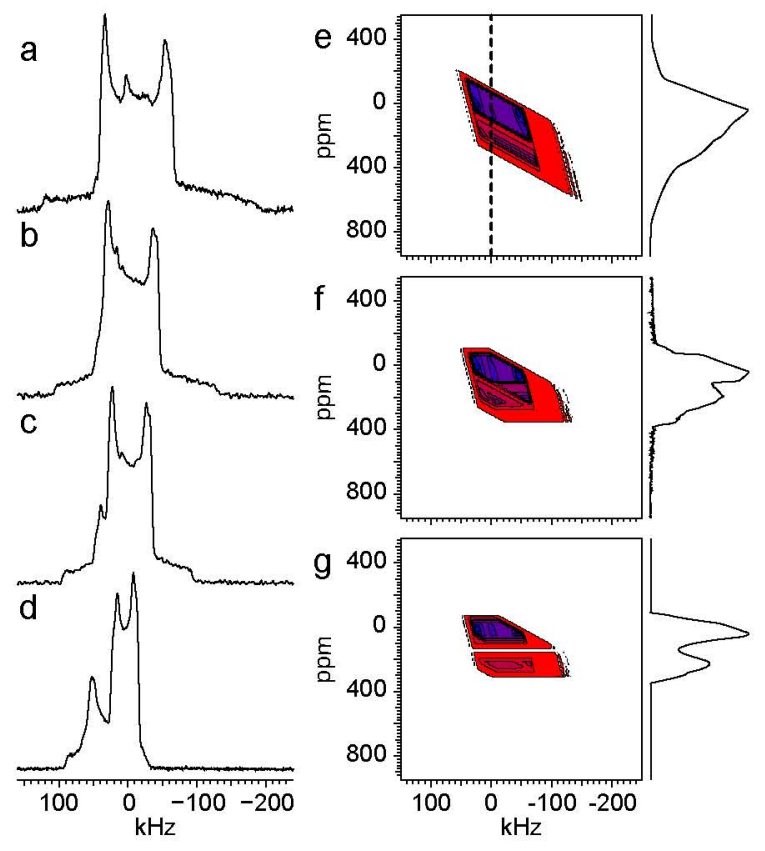

